\title{
Diagnostic Value of Adenosine-Induced Left Ventricular Diastolic Dysfunction for Detecting Coronary Artery Restenosis in Patients Undergoing Stent Implantation by Stress ECG-Gated Myocardial Perfusion SPECT - A Pilot Study -
}

Yoshimochi Nakano, MD; Naoya Matsumoto, MD; Yasuyuki Suzuki, MD; Masahiko Kato, MD; Takaaki Miki, MD; Jun Iida, MD; Shunichi Yoda, MD; Keiko Sugiyama, MD; Yuichi Sato, MD; Shu Kasama, MD; Toshio Kushiro, MD; Ken Nagao, MD; Atsushi Hirayama, MD

\begin{abstract}
Background: Usefulness of diastolic dysfunction after adenosine stress for detecting coronary stenosis has not been defined. The diagnostic accuracy of a combination of myocardial perfusion and diastolic function, as defined by prolongation of time to peak-filling rate (TTPF)/R-R and myocardial perfusion alone for the detection of coronary restenosis, was evaluated.
\end{abstract}

\begin{abstract}
Methods and Results: We used rest ${ }^{201} \mathrm{Tl} /$ adenosine stress $99 \mathrm{~m}$ Tc-tetrofosmin myocardial perfusion singlephoton emission computed tomography (SPECT) in 70 patients. Patients were divided into the following 4 groups: 20 patients with normal SPECT without stent (Control group), 20 patients showing normal SPECT without coronary restenosis (Group 1), 16 patients showing significant coronary restenosis and myocardial ischemia (Group 2a) and 14 patients showing significant coronary restenosis without myocardial ischemia (Group 2b). The TTPF, which was calculated by quantitative gated SPECT (QGS)/R-R, was not different between after stress and at rest in Control group $(0.18 \pm 0.02$ vs $0.19 \pm 0.04, P=N S)$. The TTPF/R-R after stress was significantly lower than that rest in Group $1(0.17 \pm 0.02$ vs $0.18 \pm 0.03, P<0.05)$, but TTPF/R-R after stress was significantly higher than that at rest in Groups $2 a$ and $2 b(0.22 \pm 0.03$ vs $0.16 \pm 0.03, P<0.001$ in Group $2 a$ and $0.19 \pm 0.02$ vs $0.16 \pm 0.02, P<0.001$ in Group $2 b$, respectively). Diagnostic accuracy improved from $72 \%$ to $92 \%$ when prolongation of TTPF/R-R was taken into account $(P<0.001)$.
\end{abstract}

Conclusions: Diastolic dysfunction after stress was an accurate marker for detecting significant restenosis following stent implantation. (Circ J 2010; 74: 2658-2665)

Key Words: Coronary artery disease; Coronary restenosis; Diastolic function; Stent; Time to peak filling

$\mathbf{S}$ tent implantation in percutaneous coronary intervention (PCI) was a common procedure for both stable angina and acute coronary syndrome. ${ }^{1}$ According to a previous report, implantation of a drug-eluting stent is well known to cause a low rate of coronary restenosis (approximately 8\%) following PCIs. ${ }^{2}$ However, detection of coronary restenosis after stent implantation is a still critical issue. Electrocardiogram (ECG)-gated myocardial perfusion single-photon emission computed tomography (SPECT) with exercise or pharmacological stress allows for the detection of coronary stenosis by demonstrating myocardial ischemia and regional wall motion abnormality. ${ }^{3-6} \mathrm{~A}$ recent development of quantitative gated SPECT (QGS) software allows quantitative measurement of left ventricular diastolic function as described by time to peak-filling (TTPF) using 16-frame ECG-gated SPECT. ${ }^{7,8}$ Left ventricular diastolic dysfunction is an early marker of coronary artery disease, diabetes mellitus and hypertension. ${ }^{9-11}$ Conversely, left ventricular diastolic dysfunction might improve immediately after successful PCI. ${ }^{12}$ We measured TTPF and calculated the TTPF/R-R interval in

Received February 3, 2010; revised manuscript received July 4, 2010; accepted July 27, 2010; released online October 19, 2010 Time for primary review: 25 days

Division of Cardiology, Department of Medicine, Nihon University School of Medicine, Tokyo (Y.N., N.M., Y. Suzuki, M.K., T.M., J.I., S.Y., K.S., K.N., A.H.); Department of Imaging, Health Park Clinic, Takasaki (Y.Sato); Department of Cardiovascular Medicine, Gunma University School of Medicine, Maebashi (S.K.); and Nihon University Health Planning Center, Tokyo (T.K.), Japan

Mailing address: Naoya Matsumoto, MD, Department of Cardiology, Nihon University Surugadai Hospital, 1-8-13 Kanda-Surugadai, Chiyoda-ku, Tokyo 101-8309, Japan. E-mail: matsumoto.naoya@ nihon-u.ac.jp

ISSN-1346-9843 doi:10.1253/circj.CJ-10-0095

All rights are reserved to the Japanese Circulation Society. For permissions, please e-mail: cj@j-circ.or.jp 
patients without performing PCI and with normal findings in stress myocardial perfusion SPECT (SMPS) (Control group), in patients with successful PCI (no restenosis) and with normal findings in SMPS (Group 1), in patients with coronary restenosis after PCI and with ischemic findings in SMPS (Group 2a), and in patients with coronary restenosis after PCI and without ischemic findings in SMPS (Group 2b). We evaluated whether the TTPF and TTPF/R-R values might add an incremental diagnostic value for detecting coronary restenosis with or without significant myocardial ischemia in adenosine stress myocardial perfusion SPECT.

\section{Methods}

\section{Patient Selection and Study Protocol}

The total population comprised 70 patients in whom rest ${ }^{201} \mathrm{Tl}$ adenosine stress $99 \mathrm{~m}$ Tc-tetrofosmin ECG-gated myocardial perfusion SPECT was performed. For the evaluation of diastolic function in normal subjects, we used 20 patients who had normal myocardial perfusion SPECT (10 male patients and 10 female patients). Six to 12 months after the stent implantation, 50 patients performed both adenosine stress SPECT and conventional coronary angiography. The study population includes: the Control group in whom PCI was not performed and with normal findings in SMPS $(n=20)$, Group 1 with successful PCI (no restenosis) and with normal findings in SMPS ( $n=20$ ), Group 2a with coronary restenosis after PCI and with ischemic findings in SMPS $(n=16)$ and Group $2 b$ with coronary restenosis after PCI and without ischemic findings in SMPS $(n=14)$. In Group 2, which included 30 patients, 20 patients with 1 vessel disease, 7 patients with 2-vessel disease and 3 patients with 3 -vessel disease were included. Patients in Group 2 had undergone stent implantation; 16 lesions in the left anterior descending coronary artery, 3 lesions in the left circumflex coronary artery and 11 lesions in the right coronary artery (Table 1). Patients with heart failure, apparent valvular disease, left bundle-branch block, previous coronary artery bypass surgery, paced-rhythm and frequent arrhythmias were excluded. All patients were confirmed to have no left ventricular hypertrophy by echocardiography.

\begin{tabular}{|c|c|c|c|c|c|}
\hline \multirow{2}{*}{ Stenosis } & \multicolumn{2}{|c|}{ Group 2a } & \multicolumn{2}{|c|}{ Group 2b } & \multirow{2}{*}{ Tota } \\
\hline & $76-90 \%$ & $>91 \%$ & $76-90 \%$ & $>91 \%$ & \\
\hline LAD & 6 & 3 & 5 & 2 & 16 \\
\hline LCx & 1 & 1 & 1 & 0 & 3 \\
\hline RCA & 4 & 1 & 5 & 1 & 11 \\
\hline
\end{tabular}

LAD, left anterior descending artery; LCx, left circumflex artery; $\mathrm{RCA}$, right coronary artery.

\section{Adenosine Stress Myocardial Perfusion SPECT Protocol}

All patients underwent adenosine stress dual-isotope myocardial perfusion SPECT, as previously described (Figure 1). ${ }^{13}$ First, ${ }^{201} \mathrm{Tl}(111 \mathrm{MBq})$ was injected intravenously and rest ${ }^{201} \mathrm{Tl}$ imaging was initiated $5 \mathrm{~min}$ after injection of the isotope. Patients then had adenosine stress for $6 \mathrm{~min}$. ${ }^{99 \mathrm{~m} T c-t e t r o-}$ fosmin was injected $3 \mathrm{~min}$ after the start of adenosine injection $\left(120 \mu \mathrm{g} \cdot \mathrm{kg}^{-1} \cdot \mathrm{min}^{-1}\right.$, total $\left.6 \mathrm{~min}\right)$. Stress acquisition was

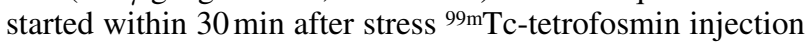
(740 MBq). Patients were asked to discontinue $\beta$-blockers for $48 \mathrm{~h}$, and nitrates, caffeine and calcium channel blockers for $24 \mathrm{~h}$ prior to the study.

\section{Acquisition Protocol and Image Reconstruction}

Rest and stress scans were acquired on a dual-detector gamma camera in the $90^{\circ}$ detector configuration (E-CAM; Siemens Medical Solutions, Erlangen, Germany), using a low-energy and high-resolution collimator, step and shoot detector rotation, 64 projections over an $180^{\circ}$ arc from the right anterior oblique (RAO) to the left posterior oblique (LPO), and a non-circular orbit. Poststress images were acquired in the supine and prone positions. Sixteen framed ECG-gated rest ${ }^{201} \mathrm{Tl}$ images were acquired for $23 \mathrm{~s} /$ projection for 64 stops ( 32 stops/head), giving an acquisition time of $16 \mathrm{~min}$. Sixteen

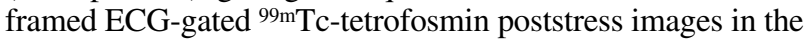
supine position were acquired for 20 s/projection ( $14 \mathrm{~min}$ ) and non-ECG-gated $99 \mathrm{~m}$ Tc-tetrofosmin prone poststress images were acquired for $18 \mathrm{~s} /$ projection $(9 \mathrm{~min})$. For prone-position

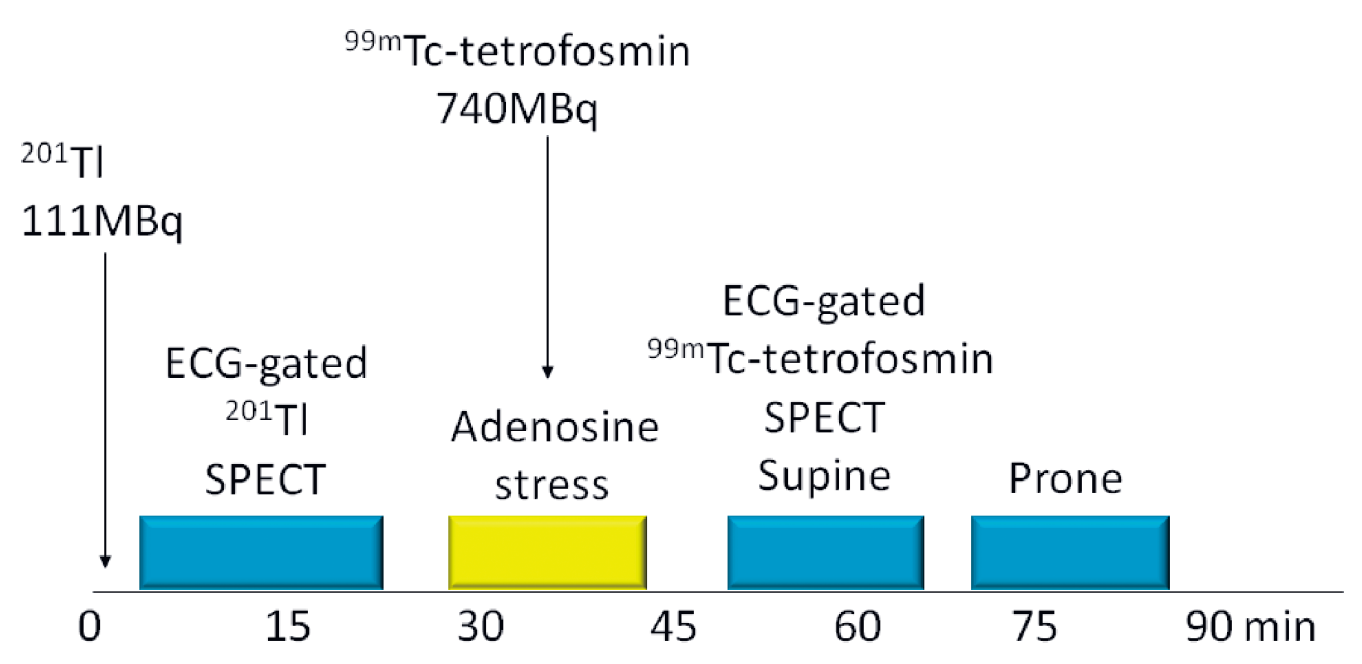

Figure 1. Rest ${ }^{201} \mathrm{Tl}$ and adenosine stress $99 \mathrm{mTC}$-tetrofosmin separate acquisition dual-isotope protocol. Patients were in the prone-position after supine-position acquisition to avoid attenuation of inferior walls. ECG, electrocardiogram; SPECT, singlephoton emission computed tomography. 


\section{Short axis}
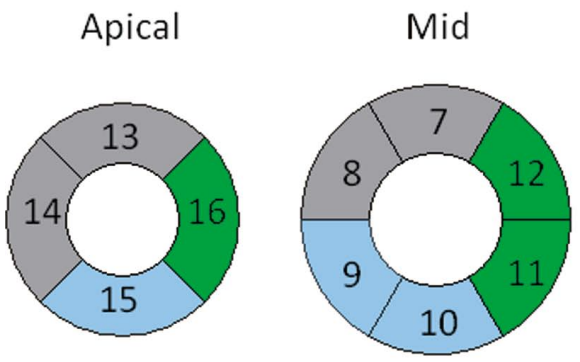

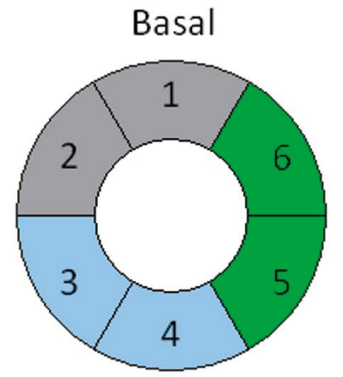

\section{Vertical long axis}

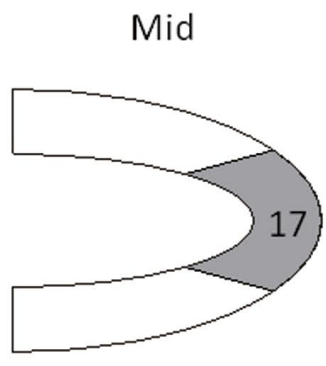

LAD (Left anterior descending artery)

LCx (Left circumflex artery)

RCA (Right coronary artery)

Figure 2. Seventeen segment model of myocardial perfusion single-photon emission computed tomography recommended by the American Heart Association.

imaging, patients lay prone on the table and the detectors rotated underneath the table from the RAO to the LPO.$^{14}$ All patients performed stress acquisitions in the supine and prone positions. Interpretation of inferior walls was considered with prone-position imaging, which can decrease the frequency of attenuation artifacts in the inferior walls. ${ }^{201} \mathrm{Tl}$ images at rest were acquired with $30 \%$ of a symmetrical window over an $80 \mathrm{KeV}{ }^{201} \mathrm{Tl}$ photopeak. All ${ }^{99 \mathrm{~m} T c-t e t r o f o s m i n ~ i m a g e s ~ w e r e ~}$ acquired with a $15 \%$ of a symmetrical window over the $140 \mathrm{keV}{ }^{99 \mathrm{~m} T c}$ photopeak. The raw projection datasets were filtered with a Butterworth filter (order 10 and cutoff frequency 0.38 cycles/cm for rest images, order 5 and cutoff frequency 0.5 cycles/cm for stress supine images, and order 5 and cutoff frequency 0.42 cycles/cm for stress prone images). Scatter or attenuation correction was not applied. Filtered raw projection images were automatically reconstructed into transverse datasets. The 3-dimensional location of the long axis of the left ventricle was determined automatically, and the short-, vertical long- and horizontal long axis images were generated.

\section{Definition of Stent Restenosis on Coronary Angiography}

All the patients underwent coronary angiography within 1 week after the SPECT scans. Initially, the angiographic results were visually interpreted by at least 2 experienced angiographers unaware of the SPECT data. Next, the percent luminal narrowing was quantitatively assessed by measuring the diameter of the region of maximum luminal narrowing and of the proximal and distal reference segments. Significant coronary artery restenosis was defined as luminal narrowing $\geq 76 \%$ of the left anterior descending artery, the left circumflex artery and the right coronary artery. Definition of coronary restenosis included in-stent and also the stent-edge (within $5 \mathrm{~mm}$ ) stenosis.

\section{Myocardial Perfusion and ECG-Gated Parameters}

Myocardial perfusion SPECT images were semiquantitatively scored by experienced readers (N.M. and Y.N.) using a 17-segment model of the ventricle with a 5-point scale system $(0=$ normal uptake, $1=$ mild hypoperfusion, $2=$ moderate hypoperfusion, $3=$ severe hypoperfusion and $4=$ no uptake). ${ }^{15,16}$
In this model, the left anterior descending artery distribution territory comprised 7 segments (segments 1,2,7,8,13,14 and 17), the left circumflex artery comprised 5 segments (segments 5,6,11,12 and 16) and the right coronary artery comprised 5 segments (segments 3,4,9,10 and 15) (Figure 2). ${ }^{17}$ Summed stress score (SSS) and summed rest score were calculated by adding the scores of 17 segments on the stress and rest images, respectively. A SSS equal to 0 was defined as a normal SPECT group. Summed difference score (SDS) was derived as the difference between the stress and rest scores. SDS $\geq 2$ was defined to be a significant inducible ischemia. In contrast, non-reversible segments (stress-rest score combinations of $4-4,4-3,3-3,3-2$, or 2-2) were judged as infarcted or non-ischemic myocardium. ${ }^{13,18}$ Discordance in image interpretation were resolved by readers' consensus.

End diastolic volume, ejection fraction (EF) and TTPF as the interval between end systole and the peak-filling rate (PFR) were automatically derived from QGS software at both rest and after stress images. Akincioglu et al showed the method that QGS software calculated the left ventricular filling rate/time curve, which computed from the first derivative of the time-volume curve. The PFR is defined as the greatest filling rate in early diastole and corresponds to the peak value of the first derivative of the diastolic portion of the timeactivity curve. TTPF is the interval between the end-systole and the PFR. ${ }^{8}$ For assessing the influence of the R-R interval to the TTPF value, we evaluated both TTPF and TTPF/R-R, which was calculated by dividing TTPF by the R-R interval on ECG. ${ }^{19}$

\section{Diagnostic Accuracy}

The sensitivity, specificity and accuracy for detecting significant coronary restenosis were calculated using either myocardial ischemia alone or the combination of myocardial ischemia and/ or left ventricular diastolic dysfunction. The definition of left ventricular diastolic dysfunction was the presence of TTPF/R-R prolongation after stress in comparison to $\mathrm{TTPF} / \mathrm{R}-\mathrm{R}$ at rest. 


\begin{tabular}{|c|c|c|c|c|}
\hline & Control $(n=20)$ & Group $1(n=20)$ & Group 2a $(n=16)$ & Group $2 b(n=14)$ \\
\hline Male & $10(50 \%)$ & $17(85 \%)$ & $15(93.7 \%)^{\star}$ & $11(78.5 \%)$ \\
\hline Age & $65.7 \pm 11.2$ & $65.2 \pm 10.4$ & $66.9 \pm 10.0$ & $65.8 \pm 10.1$ \\
\hline Hypertension & $14(70 \%)$ & $13(65 \%)$ & $10(62.5 \%)$ & $11(78.5 \%)$ \\
\hline Dyslipidemia & $13(65 \%)$ & $19(95 \%)$ & $10(62.5 \%)$ & $11(78.5 \%)$ \\
\hline Diabetes mellitus & $3(15 \%)$ & $10(50 \%)^{*}$ & $8(50 \%)$ & $8(57.1 \%)^{\dagger}$ \\
\hline Smoking & $7(35 \%)$ & $7(35 \%)$ & 7 (43.7\%) & $6(42.8 \%)$ \\
\hline Family history of CAD & $0(0 \%)$ & $1(5 \%)$ & $2(12.5 \%)$ & $0(0 \%)$ \\
\hline Previous MI & $0(0 \%)$ & $5(25 \%)$ & $8(50 \%)^{\dagger}$ & $6(42.9 \%)^{\dagger}$ \\
\hline Statin & $8(40 \%)$ & $14(70 \%)$ & $9(56.2 \%)$ & $11(78.5 \%)^{*}$ \\
\hline$\beta$-blocker & $4(10 \%)$ & $6(30 \%)$ & $8(50 \%)^{*}$ & $7(50 \%)$ \\
\hline Ca-antagonist & $10(50 \%)$ & $12(60 \%)$ & $10(62.5 \%)$ & $9(64.2 \%)$ \\
\hline ARB & $6(30 \%)$ & $11(55 \%)$ & $9(56.2 \%)$ & $8(57.1 \%)$ \\
\hline ACE-inhibitor & $3(15 \%)$ & $4(20 \%)$ & $5(31.2 \%)$ & $4(28.4 \%)$ \\
\hline Nitrate & $0(0 \%)$ & $1(5 \%)$ & $3(18.7 \%)$ & $2(14.2 \%)$ \\
\hline Antiplatelet agent & $11(55 \%)$ & $18(90 \%)^{*}$ & $14(87.5 \%)$ & $14(100 \%)^{*}$ \\
\hline
\end{tabular}

CAD, coronary artery disease; MI, myocardial infarction; ARB, angiotensin receptor blocker; ACE, angiotensin converting enzyme.

${ }^{*} \mathrm{P}<0.05$ vs Control group, ${ }^{\mathrm{t}} \mathrm{P}<0.01$ vs Control group.

\begin{tabular}{|c|c|c|c|c|}
\hline & Control $(n=20)$ & Group $1 \quad(n=20)$ & Group 2a $(n=16)$ & Group $2 b(n=14)$ \\
\hline EDV stress (ml) & $82.1 \pm 13.8$ & $85.9 \pm 20.9$ & $117.5 \pm 51.5^{\dagger}$ & $88.3 \pm 24.7$ \\
\hline EDV rest (ml) & $62.0 \pm 11.9$ & $61.6 \pm 12.6$ & $93.8 \pm 41.4^{\star}$ & $69.5 \pm 21.4$ \\
\hline EF stress (\%) & $68.9 \pm 7.04$ & $65.5 \pm 8.67$ & $57.7 \pm 10.3^{\dagger}$ & $64.2 \pm 10.9$ \\
\hline EF rest (\%) & $67.9 \pm 7.42$ & $64.6 \pm 11.5$ & $56.4 \pm 9.60^{\dagger}$ & $60.7 \pm 9.00^{*}$ \\
\hline HR stress (beats/min) & $66.0 \pm 9.36$ & $63.5 \pm 9.07$ & $62.9 \pm 8.97$ & $67.0 \pm 9.16$ \\
\hline HR rest (beats/min) & $61.4 \pm 6.67$ & $59.2 \pm 8.33$ & $59.9 \pm 9.95$ & $60.2 \pm 8.53$ \\
\hline TTPF stress (ms) & $169 \pm 18.8$ & $164 \pm 14.3$ & $209 \pm 35.5^{\dagger}$ & $170 \pm 19.6$ \\
\hline TTPF rest (ms) & $191 \pm 33.2$ & $188 \pm 18.8$ & $164 \pm 34.7^{\dagger}$ & $165 \pm 25.7^{\star}$ \\
\hline TTPF/R-R stress & $0.18 \pm 0.02$ & $0.17 \pm 0.02$ & $0.22 \pm 0.03^{\dagger}$ & $0.19 \pm 0.02$ \\
\hline TTPF/R-R rest & $0.19 \pm 0.04$ & $0.18 \pm 0.03$ & $0.16 \pm 0.03^{\dagger}$ & $0.16 \pm 0.02^{*}$ \\
\hline SDS & 0 & 0 & $9.5 \pm 6.0^{\dagger}$ & 0 \\
\hline SRS & 0 & $0.25 \pm 055$ & $1.7 \pm 2.7$ & $0.8 \pm 2.6$ \\
\hline SBP stress (mmHg) & $148.6 \pm 34.5$ & $139.8 \pm 17.5$ & $153.2 \pm 22.7$ & $139.1 \pm 21.6$ \\
\hline DBP stress $(\mathrm{mmHg})$ & $75.6 \pm 19.2$ & $77.1 \pm 24.7$ & $76.8 \pm 10.3$ & $72.2 \pm 11.1$ \\
\hline SBP rest $(\mathrm{mmHg})$ & $145.6 \pm 27.6$ & $145.1 \pm 18.8$ & $157.2 \pm 28.5$ & $153.4 \pm 22.5$ \\
\hline DBP rest $(\mathrm{mmHg})$ & $80.8 \pm 11.1$ & $78.0 \pm 12.3$ & $80.3 \pm 8.7$ & $79.1 \pm 7.95$ \\
\hline
\end{tabular}

EDV, end diastolic volume; EF, ejection fraction; HR, heart rate; TTPF, time to peak-filling; TTPF/RR, time to peak-filing divided by R-R interval; SDS, summed difference score; SRS, summed rest score; SBP, systolic blood pressure; DBP, diastolic blood pressure.

${ }^{*} \mathrm{P}<0.05$ vs Control group, ${ }^{\mathrm{P}} \mathrm{P}<0.01$ vs Control group.

\section{Statistical Analysis}

Continuous data are expressed as the mean \pm standard deviation. Comparisons were made using the Student's t-test or paired t-test, and categorical data were assessed using the chi-square test. A P value $<0.05$ was considered statistically significant. McNemar's chi-square test was used to determine the difference in the sensitivity and specificity.

\section{Results}

\section{Patient Population}

Patients' characteristics are listed in Table 2. As compared to the Control group, Group 1 included patients with diabetes mellitus more frequently, Group 2a included male patients and patients with a history of previous myocardial infarction more frequently, and Group $2 \mathrm{~b}$ included patients with diabetes mellitus and a history of previous myocardial infarction more frequently.

\section{Locations of Ischemia and Relevant Coronary Arteries}

In Group 2a, which showed myocardial ischemia and coronary restenosis, ischemic areas determined by myocardial perfusion SPECT and territories of the relevant coronary artery in which stents had been implanted, were completely matched.

\section{ECG-Gated Parameters at Rest and After Stress}

ECG-gated parameters are shown in Table 3. As compared to the Control group, Group 2a had significantly higher EDV, TTPF after stress and TTPF/R-R after stress. Group 2a also 


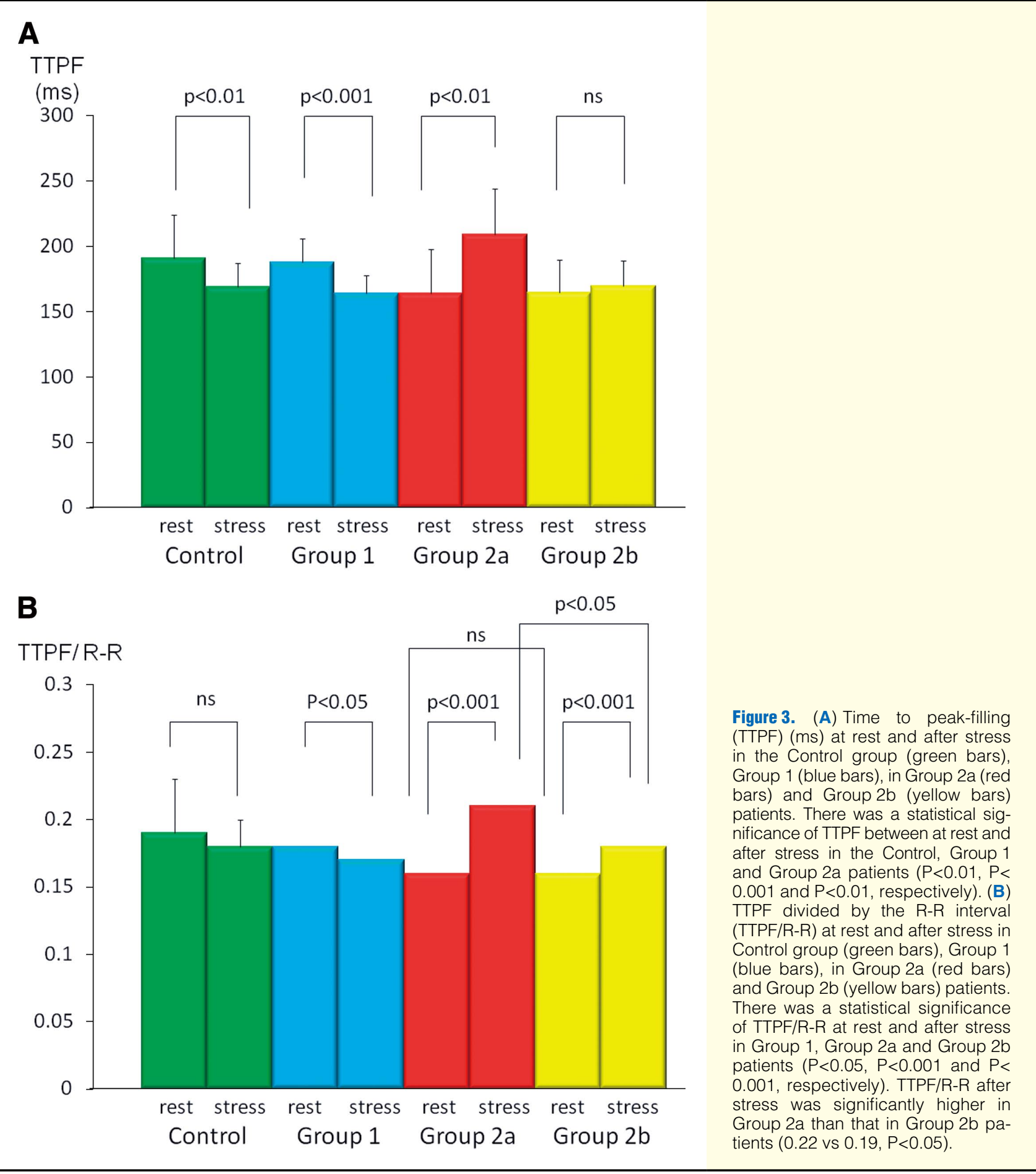

showed significantly lower EF, TTPF at rest and TTPF/R-R at rest. As compared to the Control group, Group $2 b$ had lower $\mathrm{EF}$ at rest, TTPF at rest and TTPF/R-R at rest.

Diastolic functions expressed by TTPF and TTPF/R-R are shown in Figures 3A,B. There was a statistical significance in TTPF between at rest and after stress among the Control group, Group 1 and Group 2a patients $(\mathrm{P}<0.01, \mathrm{P}<0.001$ and $\mathrm{P}<0.01$, respectively), whereas there was no significant differences in TTPF between at rest and after stress in Group $2 b$ patients $(\mathrm{P}=\mathrm{NS})$.

Figure 3B demonstrates changes of TTPF/R-R at rest and after stress among the 4 groups. The Control group revealed that TTPF/R-R between at rest and stress was comparable $(0.19 \pm 0.04$ vs $0.18 \pm 0.02, \mathrm{P}=\mathrm{NS})$. Group 1 showed a significant difference of TTPF/R-R between at rest and after stress $(0.18 \pm 0.03$ vs $0.17 \pm 0.02, \mathrm{P}<0.05)$. Group $2 \mathrm{a}$ demonstrated significant increase in TTPF/R-R after stress as compared to that at rest $(0.22 \pm 0.03$ vs $0.16 \pm 0.03, \mathrm{P}<0.001)$. Group $2 \mathrm{~b}$ also showed significant increase in TTPF/R-R at stress $(0.19 \pm 0.02$ vs $0.16 \pm 0.02, \mathrm{P}<0.001)$. Although there was no differences regarding TTPF/R-R at rest between Group 2a and Group $2 b$ patients, TTPF/R-R after stress was significantly higher in 

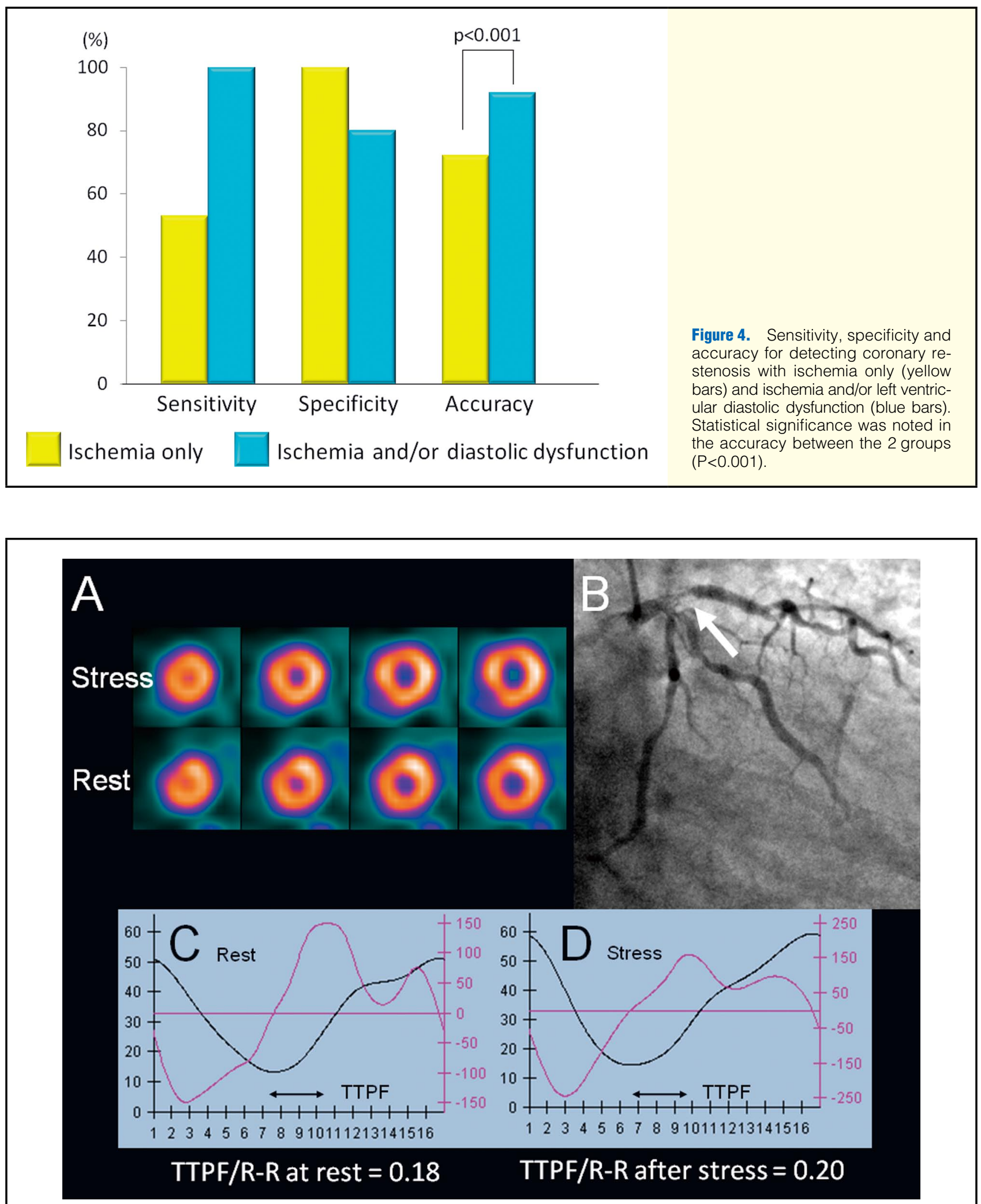

Figure 5. (A) Normal adenosine stress myocardial perfusion SPECT; (B) Significant coronary restenosis (arrow); TTPF and TTPF/R-R values at rest (C) and after stress (D) are depicted. The presence of left ventricular diastolic dysfunction was demonstrated by prolongation of TTPF/R-R ( 0.20 after stress and 0.18 at rest). SPECT, single-photon emission computed tomography; TTPF, time to peak-filling; TTPF/R-R, time to peak-filing divided by the R-R interval. 
Group $2 \mathrm{a}$ than that in Group $2 \mathrm{~b}$ patients $(\mathrm{P}<0.05)$.

\section{Diagnostic Accuracy of Coronary Restenosis}

When significant myocardial ischemia alone was used, the sensitivity and specificity for diagnosis of stent restenosis was $53 \%$ and $100 \%$, respectively. When the combination of myocardial ischemia and/or presence of left ventricular diastolic dysfunction was taken into account, the sensitivity and specificity was $100 \%$ and $80 \%$, respectively. According to McNemar's chi-square test, the diagnostic accuracy improved from $72 \%$ to $92 \%$ when the presence of left ventricular diastolic dysfunction was taken into account $(\mathrm{P}<0.001)$ (Figure 4).

\section{Representative Case}

A 74-year-old male patient who was diagnosed as having an effort angina had a stent implantation in the proximal portion of the left anterior descending coronary artery. His adenosine stress myocardial perfusion SPECT showed normal perfusion after 6 months following PCI (Figure 5A). His blood pressure was $112 / 70 \mathrm{mmHg}$ at rest and $98 / 60 \mathrm{mmHg}$ after stress. His heart rate was 73 beats/min at rest and 79 beats/min after stress. He had neither chest pain nor ECG changes during the adenosine stress test. A coronary angiography revealed $90 \%$ of coronary restenosis in the left anterior descending coronary artery (Figures 5B). Although there was no myocardial perfusion defect, TTPF/R-R after stress was higher than that at rest in this patient $(0.20$ vs 0.18$)$ (Figures $5 C, D)$.

\section{Discussion}

Systolic dysfunction of the left ventricle is frequently associated with significant coronary artery disease and it is a major determinant of patients' prognosis..$^{4,20-24}$ Homans et al noted that postischemic regional wall motion abnormalities during systole persisted only 15-30 min after exercise stress. ${ }^{25}$ Therefore, the time interval between the stress test and start of SPECT acquisition might diminish postischemic regional wall motion abnormalities. ${ }^{6}$ In contrast, left ventricular diastolic dysfunction is known as a sensitive marker of myocardial ischemia and it persists longer than systolic dysfunction. ${ }^{26-28}$ However, adenosine stress might differ from exercise stress with regard to myocardial oxygen consumption. Potential mechanisms of left ventricular diastolic dysfunction after adenosine stress are the follows: (1) coronary blood flow is redistributed away from the endocardium to epicardium layers in severe coronary stenosis; ${ }^{29,30}$ and (2) presence of coronary steal might cause true ischemia and left ventricular diastolic dysfunction in the setting of increased myocardial oxygen demand during adenosine stress. ${ }^{31}$

The efficacy of analyzing left ventricular diastolic function after stress by ECG-gated SPECT has also been demonstrated by Akincioglu et al. ${ }^{8}$ They applied PFR and TTPF using 16-frame ECG-gated SPECT. They showed that the time interval between the stress test and SPECT acquisition did not affect the TTPF value; therefore, persistent left ventricular diastolic dysfunction following a stress test might be well recognized by this technique. Consequently, in the present study, we determined: (1) the normal limit of TTPF at rest and after stress using dual-isotope SPECT protocol; and (2) the diagnostic accuracy of adenosine stress myocardial perfusion SPECT in conjunction with left ventricular diastolic function for the detection of coronary artery stent restenosis. The results showed normal values of TTPF in patients with normal myocardial perfusion SPECT (Control group), which were similar to results of previous studies by others (169 vs

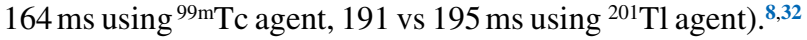
In the Control group and Group 1, the significance of TTPF between at rest and after stress might be explained by the differences in heart rate (61.4 vs 66.0 and 59.2 vs 63.5 , respectively; Table 2 and Figure 3A), but when TTPF was adjusted by the heart rate (R-R interval), the difference of TTPF between at rest and after stress in the Control group disappeared and the difference in Group 1 was diminished.

Another explanation of significance of TTPF between at rest and after stress in the Control group might have been the differences of tracers' property. ${ }^{201} \mathrm{Tl}$ causes the myocardial walls to look intrinsically thicker due to the greater Compton scatter. However, TTPF obtained from ${ }^{201} \mathrm{Tl}$ at rest is longer than that from the ${ }^{99 \mathrm{~m} T c}$ agent after stress, therefore, it does not bother the verification that there is a prolongation of TTPF after stress in cases of restenosis after STENT implantation.

Prolongation of TTPF after stress in Group 2a patients was shown in the present study and in the previous study by Nagamachi et al. ${ }^{32}$ Interestingly, the difference of TTPF between at rest and after stress in Group 2a patients was augmented when TTPF/R-R was applied $(\mathrm{P}<0.01$ in Figure 3A vs $\mathrm{P}<0.001$ in Figure 3B, red bars). Although TTPF at rest and after stress in Group $2 \mathrm{~b}$ patients did not reveal statistical significance (Figure 3A, yellow bars), the difference between TTPF/R-R at rest and after stress in Group $2 \mathrm{~b}$ patients was significant $(\mathrm{P}<0.001$, Figure $3 \mathrm{~B}$, yellow bars). The issue that TTPF/R-R is superior to TTPF in the detection of coronary artery disease has also been confirmed by Miller et al. ${ }^{19}$ The diagnostic accuracy of myocardial perfusion SPECT alone in the setting of adenosine stress for the detection of stent restenosis is relatively low with a sensitivity of $53 \%$ and a specificity of $100 \%$. The major reason for this might be an insufficient number of patients with stent restenosis. However, the diagnostic accuracy improved significantly when left ventricular diastolic dysfunction was simultaneously evaluated. In fact, accuracy to detect angiographically significant coronary restenosis in this population was $72 \%$, but when prolongation of TTPF/R-R was taken into account, the accuracy improved to $92 \%(\mathrm{P}<0.001$, Figure 4$)$. One may raise the question that the presence of previous myocardial infarction might affect left ventricular diastolic function, and it might increase the sensitivity but decrease the specificity to detect significant coronary stenosis. Actually, 14 of 30 patients in Group 2 had a history of previous myocardial infarction in the present study; all of these 14 patients showed left ventricular diastolic dysfunction after adenosine stress. However, 4 of 5 patients with previous myocardial infarction in Group 1 did not show left ventricular diastolic dysfunction. These findings suggest that the prolongation of TTPF/R-R after adenosine stress might be a reliable indicator for detecting significant coronary restenosis.

We concluded that TTPF/R-R was a more sensitive but less specific marker for detecting significant coronary restenosis following stent implantation with or without significant myocardial ischemia.

\section{Study Limitations}

First, we demonstrated differences of TTPF and TTPF/R-R among the 4 groups, but the cut-off value of TTPF or TTPF/ R-R among the 4 groups could not be defined because of the limited number of patients. Second, we evaluated global left ventricular diastolic dysfunction for detecting 1- or 2-vessel disease. However, the analysis of left ventricular regional diastolic dysfunction, which will become clinically available 
in the near future, might be more sensitive and clinically useful for the detection of stent restenosis. Finally, we asked patients to discontinue $\beta$-blockers, nitrates and calcium channel blockers, but other medications such as angiotensin receptor blockers and angiotensin-converting enzyme inhibitors might have affected diastolic function parameters.

\section{Conclusions}

In conclusion, we demonstrated that the prolongation of TTPF/R-R after adenosine stress is an accurate marker of myocardial ischemia, and it gives additional diagnostic value to myocardial perfusion SPECT alone for the detection of stent restenosis.

\section{Acknowledgments}

The authors wish to thank Mr Katsuhiko Abe for his technical assistance.

\section{References}

1. Nomoto K, Watanabe I, Oba T, Nagao K, Kushiro T, Hirayama A. Safety and efficacy of sirolimus-eluting stent in patients with acute coronary syndrome undergoing emergency procedure. Circ J 2008; 72: $1054-1058$.

2. Moses JW, Leon MB, Popma JJ, Fitzgerald PJ, Holmes DR, O'Shaughnessy C, et al. Sirolimus-eluting stents versus standard stents in patients with stenosis in a native coronary artery. $N$ Engl $J$ Med 2003; 349: 1315-1323.

3. Germano G, Kiat H, Kavanagh PB, Moriel M, Mazzanti M, Su HT, et al. Automatic quantification of ejection fraction from gated myocardial perfusion SPECT. $J$ Nucl Med 1995; 36: 2138-2147.

4. Sharir T, Berman DS, Waechter PB, Areeda J, Kavanagh PB, Gerlach J, et al. Quantitative analysis of regional motion and thickening by gated myocardial perfusion SPECT: Normal heterogeneity and criteria for abnormality. J Nucl Med 2001; 42: 1630-1638.

5. Sharir T. Role of regional myocardial dysfunction by gated myocardial perfusion SPECT in the prognostic evaluation of patients with coronary artery disease. $J$ Nucl Cardiol $2005 ; 12: 5-8$.

6. Yoda S, Sato Y, Matsumoto N, Tani S, Takayama T, Nishina H, et al. Incremental value of regional wall motion analysis immediately after exercise for the detection of single-vessel coronary artery disease: Study by separate acquisition, dual-isotope ECG-gated singlephoton emission computed tomography. Circ J 2005; 69: 301-305.

7. Nakajima K, Taki J, Kawano M, Higuchi T, Sato S, Nishijima C, et al. Diastolic dysfunction in patients with systemic sclerosis detected by gated myocardial perfusion SPECT: An early sign of cardiac involvement. J Nucl Med 2001; 42: 183-188.

8. Akincioglu C, Berman DS, Nishina H, Kavanagh PB, Slomka PJ, Abidov A, et al. Assessment of diastolic function using 16-frame 99mTc-sestamibi gated myocardial perfusion SPECT: Normal values. J Nucl Med 2005; 46: 1102-1108.

9. Boyer JK, Thanigaraj S, Schechtman KB, Perez JE. Prevalence of ventricular diastolic dysfunction in asymptomatic, normotensive patients with diabetes mellitus. Am J Cardiol 2004; 93: 870-875.

10. Yuda S, Fang ZY, Marwick TH. Association of severe coronary stenosis with subclinical left ventricular dysfunction in the absence of infarction. $J$ Am Soc Echocardiogr 2003; 16: 1163-1170.

11. Galderisi M, Cicala S, Caso P, De Simone L, D'Errico A, Petrocelli A, et al. Coronary flow reserve and myocardial diastolic dysfunction in arterial hypertension. Am J Cardiol 2002; 90: 860-864.

12. Bayata S, Susam I, Pinar A, Dinckal MH, Postaci N, Yesil M. New Doppler echocardiographic applications for the evaluation of early alterations in left ventricular diastolic function after coronary angioplasty. Eur J Echocardiogr 2000; 1: 105-108.

13. Matsumoto N, Sato Y, Suzuki Y, Kunimasa T, Yoda S, Iida J, et al. Prognostic value of myocardial perfusion single-photon emission computed tomography for the prediction of future cardiac events in a Japanese population: A middle-term follow-up study. Circ J 2007; 71: $1580-1585$.

14. Nishina H, Slomka PJ, Abidov A, Yoda S, Akincioglu C, Kang X, et al. Combined supine and prone quantitative myocardial perfusion SPECT: Method development and clinical validation in patients with no known coronary artery disease. J Nucl Med 2006; 47: 51 -
58.

15. Berman DS, Hachamovitch R, Kiat H, Cohen I, Cabico JA, Wang $\mathrm{FP}$, et al. Incremental value of prognostic testing in patients with known or suspected ischemic heart disease: A basis for optimal utilization of exercise technetium-99m sestamibi myocardial perfusion single-photon emission computed tomography. $\mathrm{J} \mathrm{Am} \mathrm{Coll}$ Cardiol 1995; 26: 639-647.

16. Matsumoto N, Sato Y, Suzuki Y, Kasama S, Nakano Y, Kato M, et al. Incremental prognostic value of cardiac function assessed by ECG-gated myocardial perfusion SPECT for the prediction of future acute coronary syndrome. Circ J 2008; 72: 2035-2039.

17. American Society of Nuclear Cardiology. Imaging guidelines for nuclear cardiology procedures, part 2. J Nucl Cardiol 1999; 6: G47G84.

18. Zellweger MJ, Dubois EA, Lai S, Shaw LJ, Amanullah AM, Lewin $\mathrm{HC}$, et al. Risk stratification in patients with remote prior myocardial infarction using rest-stress myocardial perfusion SPECT: Prognostic value and impact on referral to early catheterization. $J$ Nucl Cardiol 2002; 9: 23-32.

19. Miller TR, Grossman SJ, Schectman KB, Biello DR, Ludbrook PA, Ehsani AA. Left ventricular diastolic filling and its association with age. Am J Cardiol 1986; 58: 531-535.

20. Johnson LL, Verdesca SA, Aude WY, Xavier RC, Nott LT, Campanella MW, et al. Postischemic stunning can affect left ventricular ejection fraction and regional wall motion on post-stress gated sestamibi tomograms. J Am Coll Cardiol 1997; 30: $1641-$ 1648.

21. Ambrosio G, Betocchi S, Pace L, Losi MA, Perrone-Filardi P, Soricelli A, et al. Prolonged impairment of regional contractile function after resolution of exercise-induced angina: Evidence of myocardial stunning in patients with coronary artery disease. Circulation 1996; 94: 2455-2464.

22. Kapetanopoulos A, Ahlberg AW, Taub CC, Katten DM, Heller GV. Regional wall-motion abnormalities on post-stress electrocardiographic-gated technetium-99m sestamibi single-photon emission computed tomography imaging predict cardiac events. $J$ Nucl Cardiol 2007; 14: 810-817.

23. Petix NR, Sestini S, Marcucci G, Coppola A, Arena A, Nassi F, et al. Can the reversible regional wall motion abnormalities on stress gated Tc-99m sestamibi SPECT predict a future cardiac event? J Nucl Cardiol 2005; 12: 20-31.

24. Hida S, Chikamori T, Tanaka H, Igarashi Y, Hatano T, Usui Y, et al. Diagnostic value of left ventricular function after adenosine triphosphate loading and at rest in the detection of multi-vessel coronary artery disease using myocardial perfusion imaging. $J \mathrm{Nucl}$ Cardiol 2009; 16: 20-27.

25. Homans DC, Sublett E, Dai XZ, Bache RJ. Persistence of regional left ventricular dysfunction after exercise-induced myocardial ischemia. J Clin Invest 1986; 77: 66-73.

26. Bonow RO, Vitale DF, Bacharach SL, Frederick TM, Kent KM, Green MV. Asynchronous left ventricular regional function and impaired global diastolic filling in patients with coronary artery disease: Reversal after coronary angioplasty. Circulation 1985; 71: 297-307.

27. Wijns W, Serruys PW, Slager CJ, Grimm J, Krayenbuehl HP, Hugenholtz PG, et al. Effect of coronary occlusion during percutaneous transluminal angioplasty in humans on left ventricular chamber stiffness and regional diastolic pressure-radius relations. J Am Coll Cardiol 1986; 7: 455-463.

28. Ishii K, Imai M, Suyama T, Maenaka M, Nagai T, Kawanami M, et al. Exercise-induced post-ischemic left ventricular delayed relaxation or diastolic stunning: Is it a reliable marker in detecting coronary artery disease? J Am Coll Cardiol 2009; 53: 698-705.

29. Beller GA, Holzgrefe HH, Watson DD. Effects of dipyridamoleinduced vasodilation on myocardial uptake and clearance kinetics of thallium-201. Circulation 1983; 68: 1328-1338.

30. Becker LC. Conditions for vasodilator-induced coronary steal in experimental myocardial ischemia. Circulation 1978; 57: $1103-$ 1110.

31. Ben-Haim S, Gips S, Merdler A, Front A, Tamir A. Myocardial stunning demonstrated with rest and post-stress measurements of left ventricular function using dual-isotope gated myocardial perfusion SPECT. Nucl Med Commun 2004; 25: 657-663.

32. Nagamachi S, Wakamatsu H, Fujita S, Nishii R, Kamimura K, Kiyohara S, et al. Assessment of diastolic function using 16-frame 201Tl gated myocardial perfusion SPECT: A comparative study of QGS2 and pFAST2. Ann Nucl Med 2008; 22: 115-122. 